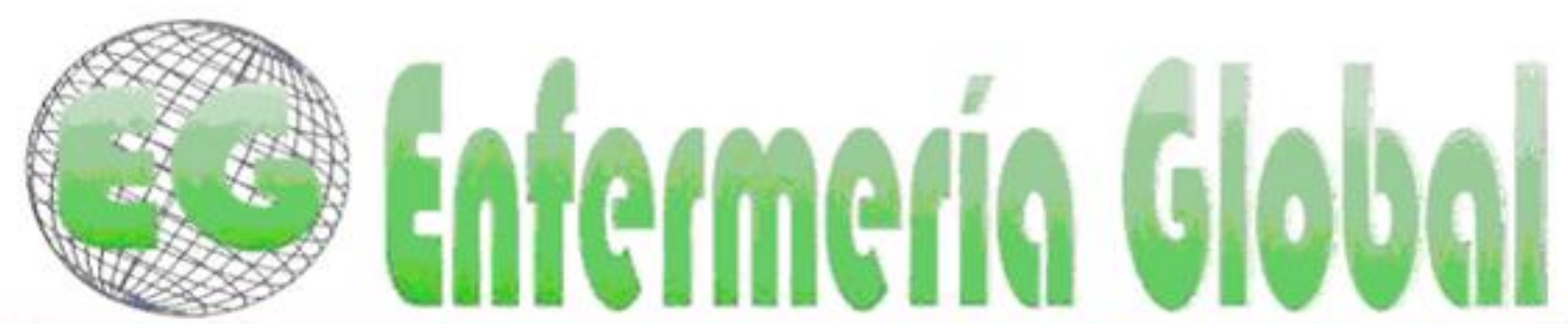

ISSN 1695-6141

Revista electrónica trimestral de Enfermeria

No38

www.um.es/eglobal/

Abril 2015

\title{
CLÍNICA
}

\section{Evaluación de los conocimientos y de la aplicación de la tetanalgesia en una unidad de neonatología}

Assessment of knowledge and application of tetanalgesia in a unit of neonatology

\section{*Casado Gómez, Cristina *Pazos Seoane, Laura *Pavón de la Maya, María José *López Jiménez, Lidia *Escobedo Mesas, Elisabeth **Bernal Herrera, Pedro}

\author{
"Residentes de Enfermería Pediátrica. E-mail: cristina.casadogomez@gmail.com **Enfermero. \\ Hospital Infantil. Hospital Universitario Virgen del Rocío. Sevilla. España.
}

\author{
Palabras Clave: Tetanalgesia; dolor; neonato; lactancia materna \\ Keywords: Tetanalgesia; pain; infant; breastfeeding
}

\section{RESUMEN}

Introducción: Durante la hospitalización, los neonatos están sometidos a constantes procedimientos dolorosos y estresantes. Está demostrado que la lactancia materna realiza un efecto analgésico. Este beneficio analgésico se conoce como tetanalgesia.

Objetivo: Valorar el conocimiento de los profesionales sanitarios sobre la tetanalgesia y analizar las causas de su falta de implantación durante los procedimientos dolorosos realizados en el neonato hospitalizado.

Material y Métodos: Se realizó un estudio observacional, transversal y descriptivo, mediante un cuestionario validado autocumplimentado por los profesionales sanitarios en la Unidad de Neonatología del Hospital Universitario Virgen del Rocío (Sevilla).

Resultados y Conclusiones: Del total de la población estudiada, 51 profesionales sanitarios, sólo el $45 \%$ conoce el concepto de tetanalgesia lo que denota el déficit de formación al respecto. De estos, sólo el $22 \%$ lo aplica. Las causas principales de la no aplicabilidad fueron la falta de consenso de los profesionales $(55,56 \%)$ y la falta de tiempo $(38,89 \%)$, factores relacionados con el trabajo de equipo y con las condiciones de trabajo.

\section{ABSTRACT}

Introduction: During the hospitalization, newborn children are subjected to constant painful and stressful procedures. There is evidence that breastfeeding makes an analgesic effect. This is known as Tetanalgesia. 
Objective: To value the knowledge of health professionals on tetanalgesia and analyze the causes of their lack of implantation during painful procedures realized to hospitalize newborn children.

Material and Methods: Was realized an observational, cross-sectional and descriptive study, using a validated questionnaire auto completed by health professionals in the Unit of Neonatology of the Hospital University Virgen del Rocío (Seville).

Results and Conclusions: Of the total study population (51 health professionals), only $45 \%$ knew the concept of tetanalgesia what it denotes the deficit of formation in the matter. Of these, only $22 \%$ apply it. The main causes of non-applicability were lack of consensus among the professionals $(55,56 \%)$ and lack of time $(38,89 \%)$,factors related to teamwork and working conditions.

\section{INTRODUCCIÓN}

Hace más de una década se pensaba que la incapacidad de los niños para verbalizar sus sentimientos y expresar su dolor era sinónimo de incapacidad para experimentarlo y recordarlo por lo que no era un motivo de preocupación para los profesionales que cuidaban a los niños prematuros ${ }^{(1)}$. Sin embargo, ahora sabemos que las estructuras anatómicas, fisiológicas y neuroquímicas que transmiten el dolor se encuentran desarrolladas antes del nacimiento ${ }^{(2)}$. Además se sabe que la vía inhibitoria descendente nociceptiva no está funcionalmente madura hasta varias semanas o meses después del nacimiento ${ }^{(3)}$. En los niños nacidos a término o pretérmino se ha demostrado una respuesta fisiológica y hormonal al dolor similar, y a menudo exagerada, si la comparamos con la de niños de mayor edad y personas adultas, con menor umbral del dolor a menor edad gestacional ${ }^{(1)}$.

Esta dificultad para reconocer el dolor ha hecho que el dolor en las unidades neonatales se trate de forma insuficiente ${ }^{(4)}$; y además se han temido los posibles efectos adversos de los analgésicos ${ }^{(5)}$. Hoy en día existen numerosas pruebas que demuestran que los neonatos son capaces de sentir el dolor ${ }^{(4)}$.

La leche materna es el alimento que recibe el neonato o el lactante y que procede de su madre. Hasta el momento, y en lo que respecta a su composición, la leche materna no ha podido ser igualada por ninguna leche modificada; la leche humana es un compuesto variable y adaptable al niño ${ }^{(6)}$.

Una de las propiedades de la leche materna, no siempre aprovechada, es su efecto analgésico; conocido como Tetanalgesia.

El mecanismo analgésico de la lactancia materna no es completamente conocido, aunque puede tener un origen multifactorial, en el que se interrelacionan aspectos de la contención, el tacto, el contacto piel con piel, la estimulación de la succión y el gusto azucarado, junto con la distracción y la inducción hormonal. La combinación de todo esto consigue una alta efectividad analgésica. Comparada con la leche artificial, la leche materna contiene una mayor concentración de triptófano, precursor de melatonina, la cual aumenta la concentración de las endorfinas beta ${ }^{(7)}$.

Además la leche materna es el procedimiento analgésico más económico, ecológico, que no tiene ningún efecto adverso para el recién nacido y que sus beneficios están demostrados a todos los niveles, tanto desde el punto de vista físico como emocional, tanto para el niño como para la madre ${ }^{(8)}$. 
Un estudio realizado en Bilbao en el año 2007, en el cual se llevó a cabo un ensayo clínico para comparar la eficacia analgésica de la lactancia materna en la toma sanguínea del talón en el recién nacido sano frente a otros procedimientos, concluyéndose que el amamantamiento es el procedimiento más efectivo mostrando una reducción del tiempo de llanto del $98 \%$ frente al resto de técnicas analgésicas ${ }^{(2)}$. Una revisión Cochrane traducida en 2008 en la cual se analizan todos los ensayos clínicos que evalúan los efectos de la lactancia o de la leche materna durante un procedimiento doloroso desde los años 1966 hasta 2006, concluyen que en los neonatos la lactancia se asoció con una reducción de los cambios de la frecuencia cardíaca, la duración del llanto, el porcentaje de tiempo del llanto y de la mejoría en medidas de dolor convalidadas y no convalidadas cuando se comparó con placebo, ninguna intervención o cambio de posición ${ }^{(7)}$.

A pesar de todo lo comentado anteriormente todavía el manejo del dolor en los recién nacidos está muy lejos de ser óptimo. La prevención y tratamiento del dolor debería considerarse como un derecho humano esencial de los recién nacidos independientemente de sus consecuencias a corto o largo plazo.

El estudio de este problema viene motivado por las consecuencias negativas tanto a nivel físico como psicológico en el neonato, que se derivan de las múltiples experiencias dolorosas durante su cuidado y la necesidad de encontrar alternativas no farmacológicas eficaces e inocuas para el control del dolor. Los resultados obtenidos, posibilitarán la aplicación de medidas de mejora para lograr una mayor calidad de la práctica clínica.

Se pretende valorar el conocimiento de los profesionales sanitarios sobre la Tetanalgesia y analizar las causas de su falta de implantación durante los procedimientos dolorosos realizados en el neonato hospitalizado.

\section{MATERIAL Y MÉTODOS}

El estudio se realizó en la Unidad de Gestión Clínica de Neonatología, en la que se incluyen las plantas de Hospitalización, Cuidados Intermedios y Unidad de Cuidados Críticos Neonatales, del Hospital Universitario Virgen del Rocío de Sevilla.

Se realizó un cuestionario validado y se difundió por los profesionales de las diferentes disciplinas que desearon participar en el estudio. El cuestionario titulado "Evaluación de los conocimientos y de la aplicación de la tetanalgesia por el profesional sanitario en una unidad de neonatología", consta de cuatro apartados. En el primero de ellos se recoge información para conocer el perfil de los profesionales sanitarios. En el segundo, denominado "conocimientos", se hace un total de 10 preguntas cerradas (sí, no, no sabe), entre ellas preguntas falsas (3) y verdaderas (7), lo cual nos permitió valorar al profesional que conoce el concepto realmente y aquel que no lo conoce. El apartado tercero hace referencia a la "aplicabilidad" donde se evalúa si se realiza tetanalgesia o no y se ofrece una lista de causas de la no puesta en marcha de la tetanalgesia en caso de que la respuesta sea negativa. Por último, el apartado cuatro trata de la "formación", si es necesaria o no formación sobre el tema, si existe formación y si estaría interesado en acudir.

La población de estudio fueron los profesionales sanitarios de las diferentes disciplinas que trabajan o se forman actualmente dentro de la Unidad Clínica de Neonatología del Hospital Universitario Virgen del Rocío (Sevilla), 
independientemente de su tiempo trabajado en pediatría y, más concretamente, neonatología. Los profesionales sanitarios que desearon participar estaban informados y aceptaron bajo su responsabilidad y firma, el consentimiento informado para la participación en el presente estudio.

El análisis de los datos se llevó a cabo por el grupo de trabajo del estudio. Se extrajeron los porcentajes de cada uno de los apartados y se realizaron comparativas entre los diferentes resultados y variables.

\section{RESULTADOS}

\section{Datos personales}

- Sexo: El 15,7\% de los profesionales encuestados eran hombres (8 de 51) y el 84,31 $\%$ eran mujeres (43 de 51 ).

- Edad: El 23,53\% comprendían las edades de 20-30 años, el 25,49\% tenían entre 31 40 años, el 33,33\% entre 41-50 años, el 15,68\% entre 51-60 años y el 2\% poseían una edad mayor de 60 años.

- Tiempo trabajado en pediatría: El 17,65\% indicaban haber trabajado menos de un año en pediatría, el 41,18\% entre 1-5 años, el 19,61\% de 6-10 años trabajados, el $11,76 \%$ entre $11-20$ años y un 7,84\% había trabajado entre 21-30 años.

- Unidades clínicas en las que han trabajado en pediatría: El 86,27\% han trabajado en unidades de neonatología, el $27,45 \%$ en nefroneuropediatría, el $37,25 \%$ en medicina interna infectología pediátrica, el $27,45 \%$ en unidades de cuidados intensivos pediátricos, el $58,82 \%$ en unidades de cuidados intensivos neonatales, el $27,45 \%$ en cirugía pediátrica, el $25,49 \%$ en oncohematología pediátrica, el $45,10 \%$ en observación-urgencias pediátricas, el $21,57 \%$ en consultas externas, el $35,29 \%$ han trabajado en la unidad de lactantes y el $13,72 \%$ han trabajado en otras unidades en pediatría.

- Puesto de trabajo actual: El 47,06\% eran enfermeras, el 11,76\% eran residentes de enfermería pediátrica, el $1,96 \%$ eran estudiantes de enfermería, el $21,57 \%$ eran auxiliares de enfermería, el 3,92\% eran matronas, otro 3,92\% eran residentes de matrona y el $5,88 \%$ eran médicos residentes de pediatría.

\section{Conocimientos}

El $50 \%$ de las enfermeras conocen el concepto, el $27 \%$ de las auxiliares de enfermería, el 33\% de los médicos residentes de pediatría. De los profesionales de matrona que participaron ninguno conocía el concepto al igual que los residentes de matrona. Todos los residentes de enfermería pediátrica y los estudiantes de enfermería conocían el concepto de tetanalgesia. 


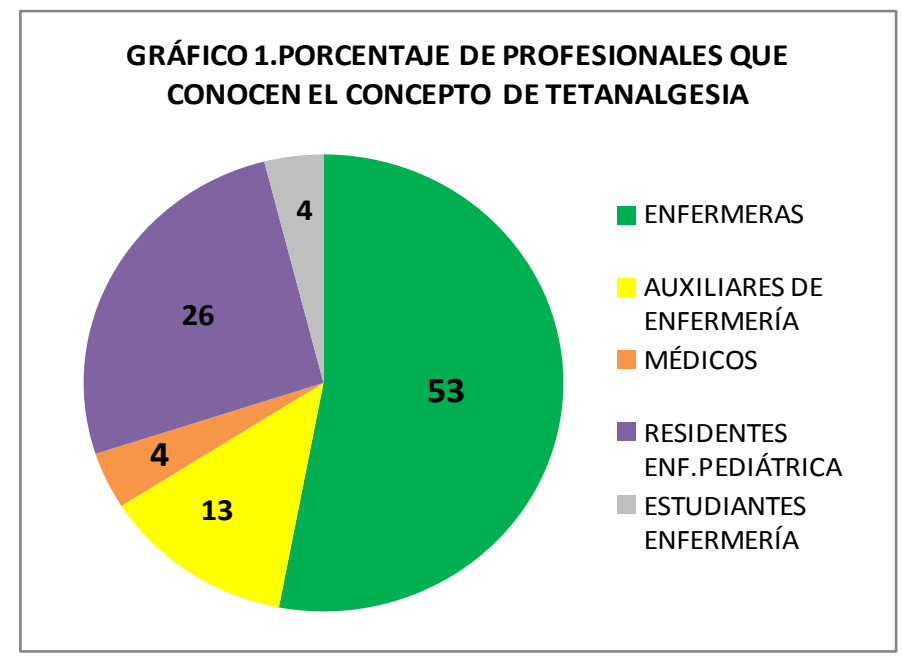

\section{Aplicabilidad}

Del total de la muestra del estudio que conoce el término de tetanalgesia (45\%), solamente el $22 \%$ de ellos utiliza en su práctica diaria la tetanalgesia. El $55 \%$ restante de los profesionales que conocen el término no lo aplican. Las razones de la no puesta en práctica fueron: falta de consenso entre los profesionales (55,56\%), falta de tiempo $(38,89 \%)$, desconocimiento $(33,34 \%)$, falta de experiencia $(33,34 \%)$, administración de sacarosa como procedimiento analgésico (27,78\%), factores ambientales $(22,23 \%)$, falta de seguridad $(11,11 \%)$ y rechazo por la presencia de los padres $(11,11 \%)$.

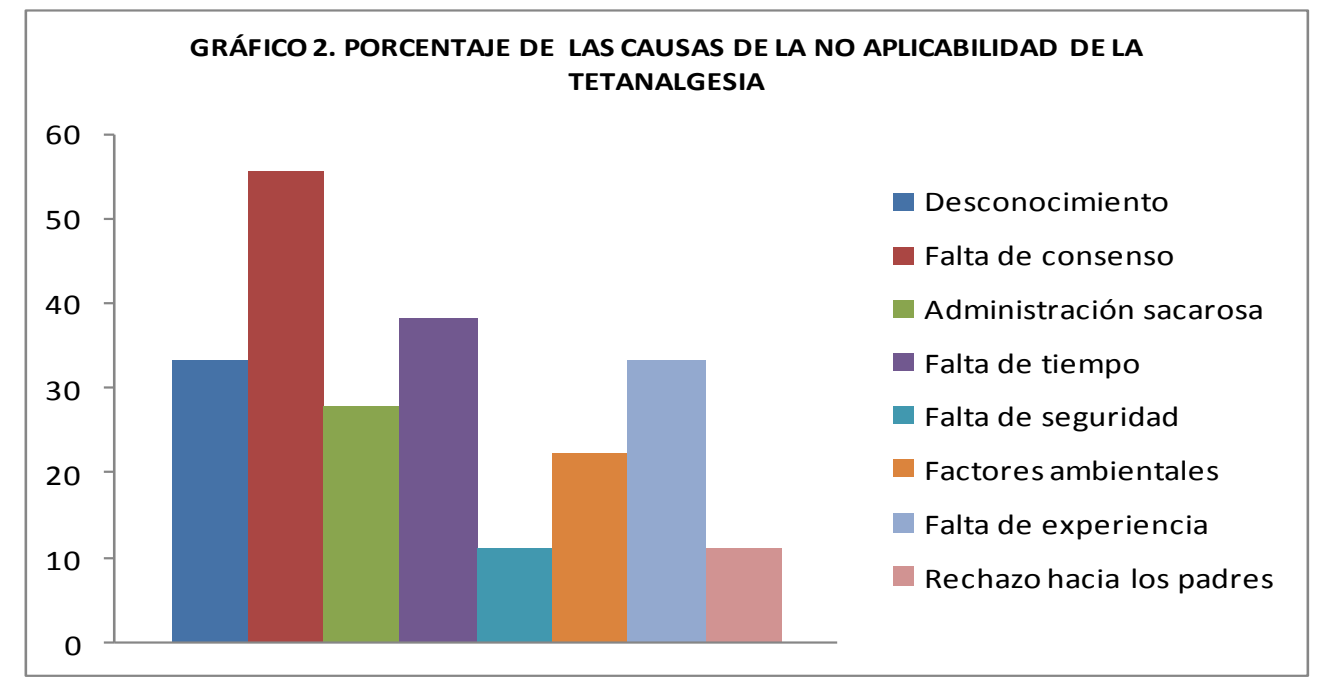

\section{Formación}

El $23,53 \%$ de los profesionales afirma que en su unidad o en su hospital realizan cursos relacionados con la tetanalgesia, el 27,45\% refiere haber asistido a formación sobre el tema aunque de estos sólo el $64,28 \%$ conoce el concepto de tetanalgesia. El 
$88,23 \%$ de los profesionales sanitarios están interesados en recibir formación sobre la tetanalgesia.

\section{DISCUSIÓN}

A pesar de las evidencias actuales que demuestran que los recién nacidos poseen la capacidad de percepción del dolor ${ }^{(1-4)}$ y los conocimientos que poseemos sobre los beneficios y las cualidades analgésicas de la lactancia materna ${ }^{(6-8)}$, nuestros resultados apuntan a que el cambio de actitud que se requiere de los profesionales sanitarios para conseguir la valoración y tratamiento del dolor en el recién nacido, está aún en un proceso lento donde día a día priman más las costumbres que la evidencia científica. Este cambio de mentalidad hacia la calidad de nuestros cuidados debe conseguirse mediante una buena base de formación para aunar los conocimientos y así evitar la diversidad en cuidados.

Podemos decir que muy próximo a la mitad de la población de estudio (45\%) conocen el efecto analgésico que posee la lactancia materna, pero sin embargo, la mayoría optan por la no realización de dicha técnica relacionándolo con motivos de trabajo en equipo y condiciones del puesto de trabajo. La causa principal señalada por los profesionales fue la "falta de consenso entre los profesionales", causa que se observa en muchas otras técnicas en el ámbito sanitario, no todos los profesionales médicos prescriben igual, no todos los profesionales de enfermería canalizan vías periféricas del mismo modo,...son ejemplos de una realidad que se observa en nuestros hospitales donde no existe una base de conocimientos evidenciados científicamente y no existe una formación adecuada para asegurar la homogeneidad de los cuidados con lo que aumentaríamos la seguridad de los profesionales para realizar sus actos, la seguridad del paciente al recibir unos cuidados evidenciados de calidad y la seguridad de los familiares al recibir y observar la misma información y cuidados a manos de los diferentes profesionales; es decir, todo esto se resume en potenciar el trabajo en equipo, en fomentar un ambiente fructífero de trabajo donde toda opinión cuente y que todo el equipo reme hacia el mismo fin, el paciente. Sin embargo, puede darse el caso de que sí exista realmente esa formación o que haya gente emprendedora y luchadora que intente darse voz dentro del grupo de trabajo pero se enfrenta a un grupo de profesionales desmotivados o inmersos en una rutina de trabajo de difícil modificación donde las personas nuevas que van entrando en el grupo o se deciden a hacer lo que mejor crean a nivel individual o bien acaban haciendo lo que "hace todo el mundo", con lo cual se entraría en un círculo vicioso donde el único perjudicado es el paciente.

La segunda causa más señalada por los profesionales fue "la falta de tiempo". Actualmente se está viviendo una dura realidad dentro de la sanidad donde con menos personal sanitario se pretende abarcar el mismo número de trabajo y la misma efectividad en los cuidados. Nos encontramos con profesionales sanitarios agobiados que no disfrutan con lo que hacen y no se llevan a su casa un "buen sabor de boca" de que han hecho todo lo que han podido en su jornada laboral. Todos conocemos la realidad que nos acecha y que esta "falta de tiempo" en muchas ocasiones es real y que por tanto, una técnica como es la tetanalgesia, que requiere de cierto tiempo (mínimo 5 minutos previos amamantando), puede resultar no prioritaria cuando tienes un límite de tiempo para realizar una serie de cuidados.

Siguiendo del orden, en el mismo nivel nos encontramos "desconocimiento" y "falta de experiencia"; es decir, no realizan tetanalgesia porque no disponen de formación o 
información suficiente para realizarla y como no la realizan no se establece la evidencia fiable y palpable que le otorga la experiencia propia.

La administración de sacarosa es la siguiente causa, la cual es otra técnica de efecto analgésico evidenciado en todos los ensayos revisados en los cuales la lactancia no tuvo ventajas cuando se comparó con concentraciones mayores de glucosa/sacarosa ${ }^{(7)}$. Por otro lado, la efectividad de la sacarosa con respecto a la lactancia se debe probablemente a la mayor concentración del azúcar en la primera, aunque el mecanismo de acción de la sacarosa tampoco es claro ${ }^{(7)}$. Es decir, con la administración de sacarosa disponemos de una acción analgésica más rápida (sólo requiere dos minutos previos), pero no existen estudios científicos que demuestren la acción que realiza la sacarosa en el neonato y la concentración mínima para producir analgesia, pudiendo cometer el error de sobrecargar con glucosa al recién nacido sin conocer la capacidad realmente existente de metabolizarlo y sintetizarlo. Por tanto, las propiedades fisiológicas, químicas y el vínculo existente madre-hijo hacen de la lactancia materna el método de elección frente a todas las técnicas existentes.

Por último nos encontramos con las causas de factores ambientales, que se pueden relacionar con lo comentado anteriormente del ambiente de trabajo y las condiciones de trabajo; falta de seguridad, por la falta de experiencia y conocimientos sobre tetanalgesia y rechazo hacia los padres. Esta última causa nos sorprendió que saliera reflejada dentro de las posibles causas de la no puesta en práctica de la tetanalgesia. A muchos profesionales les "incomoda" la presencia continua de los padres creyendo quizás, bajo nuestro punto de vista, que estos son meros agentes que buscan el más mínimo fallo a cometer por el profesional o agentes que demandan sin cesar cuidados en su hijo. Cierto es que existe mucha variabilidad en las conductas presentes de los padres y en las culturas y valores que puedan tener, lo que sí es cierto ante todo, que lo mejor para el recién nacido es la presencia de sus padres y el iniciar lo más precoz posible el vínculo afectivo con ellos, tan es así, que se considera uno de los derechos fundamentales del niño. Por tanto, no es más que emprender la difícil iniciativa de romper con la dura rutina tradicional de que los niños cuando ingresaban eran responsabilidad del profesional que los cuidaba y los padres quedaban en un segundo plano y seguir construyendo la presente realidad donde el hecho de estar ingresado es una etapa más de la vida del niño, etapa que debe vivir en primera plana junto con sus padres, los cuales deben ser agentes activos en cuidados y partícipes en todo lo relacionado con la vida y enfermedad de su hijo.

A lo largo del desarrollo del proyecto de investigación se nos plantean los siguientes problemas: en cuanto a tiempo, observamos que el cuestionario requiere de un tiempo excesivo en comparación que el tiempo que disponen los profesionales para contestarlo, limitando sus posibles respuestas y pudiendo llevar a sesgos de información. Se podría también haber inducido a un sesgo de información al otorgar a los profesionales las respuestas a las posibles causas de la no aplicación de la tetanalgesia directamente, pudiendo haber obtenido mayor información a través de preguntas abiertas. Debemos, por tanto, reevaluar nuevamente el cuestionario y otorgarle un formato adaptado a las condiciones y al personal de la unidad.

Como futuras medidas de mejora sería implantar un programa de formación para profesionales sanitarios mediante sesiones clínicas donde se hable del efecto analgésico de la lactancia materna, la tetanalgesia, así como de otros temas de relevancia clínica. Dicha formación también podría ampliarse al ámbito familiar, formando desde atención primaria en el periodo perinatal a los futuros padres sobre 
todos los aspectos de la lactancia materna, incluido la tetanalgesia, de esa manera en casos como la vacunación infantil, ya sean las propias madres las que estén previamente amamantando antes de entrar en la sala y así reduciríamos el tiempo de espera y, más importante, el dolor del niño. El valorar y aliviar el dolor debe ser objetivo fundamental dentro de una unidad de neonatología, siendo necesario nuevas investigaciones centradas en la valoración del dolor en el neonato y en la lactancia materna y sus beneficios.

Finalmente creemos que mediante este estudio hemos cumplido el objetivo planteado en los inicios de la investigación, exponiendo mediante evidencia la clara necesidad de formación en tetanalgesia y analizando las posibles causas que promueven su no puesta en práctica relacionándolas con nuestra realidad clínica.

\section{CONCLUSIONES}

Dentro del contexto de seguridad del paciente, nosotros como profesionales sanitarios tenemos el deber de garantizar el bienestar del paciente e impedir en la medida de lo posible que sienta dolor. Debemos hacer llegar a todos los profesionales en salud que la Tetanalgesia resulta ser un método inocuo, fácil y económico que nos ayudará a conseguir dicho bienestar tanto para el niño como para la familia, sobre todo la madre, fomentando nuevas investigaciones para mejorar las presentes y solventando las limitaciones que pudieran existir en cualquier unidad de trabajo para su puesta en marcha.

\section{Agradecimientos}

Agradecemos a todo el personal sanitario de la Unidad de Gestión Clínica de Neonatología del Hospital Universitario Virgen del Rocío de Sevilla por su colaboración y ayuda para la recopilación de los datos, a la supervisión de la unidad por permitir la realización del estudio observándose con ello la iniciativa de mejora existente y, por supuesto, gracias a nuestros tutores que nos guían en nuestro día a día formativo como residentes de enfermería pediátrica.

\section{BIBLIOGRAFÍA}

(1). Ministerio de Sanidad y Política Social. Revisión Bibliográfica: Cuidados desde el nacimiento. Recomendaciones basadas en pruebas y buenas prácticas. Madrid.2010. (2). Saitualturriaga,G; AguirreUnceta-Barrenechea, A; Suarez Zarate, K; Zabala Olaechea, I ; Rodríguez Núñez, A; y Romera Rivero, M. Efecto analgésico de la lactancia materna en la toma sanguínea del talón en el recién nacido. An Pediatr(Barc).2009;71(4):310-313.

(3). Anand, KJS; Hickey, PR. Pain and its effects in the human neonate and feto. N Engl J Med. 1987; 73:1-9.

(4). Anand, KJS. Consensus statement for the prevention and management of pain in the newborn. Arch Pediatr Adolesc Med. 2001; 155: 173-180.

(5). Perrault, T; Fraser-ASkin, D; Liston, R. Pain in the neonate. Pediatr Chil Health. 1997;2: 201-209.

(6). Aguilar Cordero, MJ. Conceptos, definiciones e historia de la lactancia. Nodrizas.Lactancia Materna. Madrid: Elsevier; 2005. p. 1-14.

(7). Shah, PS; Aliwalas, LL; Shah, V. Lactancia o leche maternal para los procedimientos dolorosos en los neonatos. (Revisión Cochrane traducida). En: La 
Biblioteca Cochrane Plus, 2008 Número 2. Oxford: Update Software Ltd. Disponible en: http://www.update-software.com. (Traducida de The Cochrane Library, 2008 Issue 2. Chichester, UK: John Wiley \& Sons, Ltd.).

(8). Urzainqui Zabalza, O; Caravaca Hernández, A; Egea Zerolo, B; González Álvarez, M. Revisión bibliográfica: efecto analgésico de la lactancia materna en recién nacidos a término. EG. 2004 may; (4): 1-5.

Recibido: 20 de abril 2014; Aceptado: 27 de junio 2014 